Review Article

\title{
The Effect of Acupuncture on Glucose Metabolism and Lipid Profiles in Patients with PCOS: A Systematic Review and Meta-Analysis of Randomized Controlled Trials
}

\author{
Ruqun Zheng, ${ }^{1}$ Peng Qing, ${ }^{2}$ Mei Han, ${ }^{3}$ Jinlong Song, ${ }^{4}$ Min Hu $\mathbb{D}^{1},{ }^{1}$ Hongxia Ma, ${ }^{1}$ \\ and Juan $\mathrm{Li} \mathbb{D I D}^{1}$ \\ ${ }^{1}$ Department of Traditional Chinese Medicine, The First Affiliated Hospital of Guangzhou Medical University, Guangzhou, China \\ ${ }^{2}$ Department of Acumoxibustion, The First Affiliated Hospital of Jinan University, Guangzhou, China \\ ${ }^{3}$ Beijing University of Chinese Medicine, Beijing, China \\ ${ }^{4}$ Department of Laboratory, The First Affiliated Hospital of Guangzhou Medical University, Guangzhou, China
}

Correspondence should be addressed to Juan Li; lijua@163.com

Received 8 January 2021; Revised 3 March 2021; Accepted 9 March 2021; Published 22 March 2021

Academic Editor: Yong Wang

Copyright ( $\odot 2021$ Ruqun Zheng et al. This is an open access article distributed under the Creative Commons Attribution License, which permits unrestricted use, distribution, and reproduction in any medium, provided the original work is properly cited.

Objective. To evaluate the effectiveness of acupuncture on glucose metabolism and lipid profiles in patients with polycystic ovary syndrome (PCOS). Methods. Databases, including the China National Knowledge Infrastructure (CNKI), the China Science and Technology Journal Database (VIP), Wanfang, PubMed, and the Cochrane Library were searched for the relevant literature, with the retrieval deadline being February 2020. Two reviewers independently screened, selected, and extracted the data and validated the results. The methodological quality of the included studies was evaluated with the risk of bias tool, and the meta-analysis was performed using the RevMan 5.3.5 software. Results. A total of 737 patients with PCOS from 10 randomized controlled trials were included in the metaanalysis. A pooled analysis showed significant decreases in body mass index (mean difference $(\mathrm{MD})=-1.47,95 \% \mathrm{CI}-2.35$ to -0.58 , $P<0.001)$ and waist-to-hip ratio $(\mathrm{MD}=-0.04,95 \% \mathrm{CI}[-0.06,-0.02], P<0.001)$ in the acupuncture group along with significant improvements in fasting plasma glucose $(\mathrm{MD}=-0.38,95 \% \mathrm{CI}[-0.70,-0.07], P=0.02)$, homeostasis model assessment of insulin resistance $(\mathrm{MD}=-0.22,95 \% \mathrm{CI}[-0.41,-0.02], P=0.03)$, and triglycerides $(\mathrm{MD}=-0.26,95 \% \mathrm{CI}[-0.48,-0.04], P=0.02)$. No significant differences were observed in the Ferriman-Gallwey score, $2 \mathrm{~h}$ fasting plasma glucose, fasting insulin, $2 \mathrm{~h}$ fasting insulin, serum total cholesterol, low-density lipoprotein cholesterol, or high-density lipoprotein cholesterol. Conclusion. Acupuncture is relatively effective and safe in improving glucose metabolism and insulin sensitivity in patients with PCOS. The included studies were generally of not bad methodological quality, but further large-scale, long-term randomized controlled trials with rigorous methodological standards are still warranted.

\section{Introduction}

Polycystic ovary syndrome (PCOS) is a complex endocrine disorder in reproductive-age women with an incidence of $6 \%$ to $21 \%$ globally and $5.6 \%$ to $11.2 \%$ in Chinese women [1]. PCOS generally manifests as hyperandrogenism, oligoanovulation, and/or polycystic ovaries [2], and patients with PCOS are at an increased risk for metabolic disturbances such as insulin resistance (IR), impaired glucose tolerance (IGT), dyslipidemia, insulin-induced metabolic syndrome predisposing them to type 2 diabetes mellitus (T2D), and cardiovascular disease [3-5]. Furthermore, hyperinsulinemia is considered to be associated with the mechanism of ovulatory dysfunction in women with PCOS [6].

IGT, obesity, and T2D are more prevalent in women with PCOS women than in the general population [6-8], and it is estimated that among obese women with PCOS about 30\%$40 \%$ are diagnosed with IGT and $5-10 \%$ suffer from T2D $[9,10]$. Moreover, metabolic syndrome is three times more frequent in women who present with classic PCOS symptoms [11]. The metabolic disturbances present in women with PCOS not only involve an increased risk for cardiovascular disease but 
also might worsen many of the typical PCOS symptoms, and thus they have become an important target for therapy [12]

Lifestyle measures, including diet, exercise, and behavioral modification, are the first-line treatments in obese women with PCOS for improving metabolic parameters and endocrine abnormalities [13]. Nevertheless, lifestyle changes might not be easy to sustain and might not be sufficient for significant weight loss. Thus, pharmaceutical methods targeting both reproductive and metabolic disorders might be needed.

Biguanides (metformin) and thiazolidinediones (rosiglitazone and pioglitazone) are insulin-sensitizing agents that are currently in clinical use [8]. Metformin has a few safety concerns and is the most widely used insulin-sensitizing agent for treating women with PCOS of reproductive age $[6,14]$. Metformin is an oral antihyperglycemic biguanide drug that enhances glucose uptake in skeletal muscle and adipocytes by increasing insulin sensitivity, and it is often used as a first-line pharmacological treatment for women with PCOS presenting with IR [15-17]. However, gastrointestinal symptoms like diarrhea and abdominal discomfort are common adverse effects of metformin $[18,19]$. In addition, rosiglitazone has been withdrawn from the market in many countries due to concerns of increased risk of congestive heart failure [20].

Pharmacological approaches are often effective, but there are issues with adverse effects and patient compliance due to the prolonged treatment required by PCOS patients [19]. Thus, additional nonpharmacological treatment strategies such as acupuncture should be considered in treating PCOS [19]. Acupuncture, which has been used to treat diseases for more than 2500 years in China [21], has become increasingly popular worldwide in recent years for its convenience and low incidence of adverse effects. Acupuncture is widely applied in clinical practice for treating PCOS [19], and studies have concluded that acupuncture is effective against metabolic disturbances associated with IR such as overweight [22], hyperglycemia [23, 24], and hyperlipidemia [25] by improving insulin sensitivity [26]. It has also been reported that acupuncture may improve glycemic outcomes in women with PCOS [27]. Our prospective pilot study [28] showed that homeostasis model assessment of insulin resistance (HOMA-IR) was decreased after 6 months of treatment with acupuncture, and HOMA-IR remained significantly decreased at 3 months of follow-up. In addition, electroacupuncture has been shown to decrease HOMA-IR and improve IR in a rat model of PCOS [29]. However, the designs of these studies were all considerably different and thus it is difficult to draw strong conclusions, and the effects of acupuncture on glucose and lipid metabolism in PCOS have not been systematically analyzed.

To fill this knowledge gap, this systematic review aimed at comparing the effectiveness of acupuncture to that of standard therapy (lifestyle management or insulin-sensitizing agents) in the treatment of metabolic dysfunction in patients with PCOS.

\section{Methods and Materials}

2.1. Eligibility Criteria. We only included randomized controlled trials (RCTs) using accepted interventions involving acupuncture (manual acupuncture or electroacupuncture) alone, with unrestricted acupoints or intensity, compared with placebo (placebo or sham acupuncture) or with standard therapy (lifestyle management, including weight reduction by diet and exercise or insulin-sensitizing agents such as thiazolidinediones and metformin). The subjects were adult patients with PCOS. Only studies with the outcomes of HOMA-IR, HbA1c, glucose, and insulin levels or lipid profiles were included.

2.2. Literature Search. We searched for published literature in the China National Knowledge Infrastructure (CNKI), Wanfang, the China Science and Technology Journal Database (VIP), PubMed, and the Cochrane Library databases. We also retrieved the completed but unpublished studies from the clinicaltrials.gov website and tracked the results of these studies. Only Chinese and English articles were retrieved, and the last search was carried out on 29 February 2020. The search words included "polycystic ovary syndrome", "PCOS", "acupuncture", "electroacupuncture", "needle", "needling", "scalp acupuncture", "abdominal acupuncture", "ear acupuncture", "wrist-ankle acupuncture", "warm acupuncture-moxibustion", "homeostatic model assessment", "HOMA", "glucose", "insulin", "insulin sensitivity", "metabolic", "glycemic control", "OGTT", "lipid profile", "HbA1c", "triglycerides (TG)", "total cholesterol (TC)", "high-density lipoprotein cholesterol (HDL-C)", "low-density lipoprotein cholesterol (LDL-C)", "randomized controlled trial", "clinical trial", "RCT", "random", "randomize", and "randomization". Depending on the characteristics of different databases, search strategies including both subject words + free words and keywords + full text were used.

2.3. Literature Screening. The identified articles were initially imported into NoteExpress, and the initial screening was performed based on the inclusion/exclusion criteria after reading the titles and abstracts. In the next step, full-text articles were acquired and checked for eligibility prior to including them in the final analysis. All duplicated articles and any papers that did not meet our inclusion criteria were excluded.

2.4. Data Extraction. A data extraction table was designed to collect the data to be analyzed, including the following aspects: (a) basic characteristics of the included studies; (b) research methods and possible biases; (c) participant characteristics; (d) interventions; (e) outcome measures; (f) research findings; and $(\mathrm{g})$ other required information.

Two investigators (Zheng and Qing) independently extracted the data and assessed the quality of all relevant RCTs. The following data were extracted from the published RCTs: the first author's name, year of publication, the country where the trial was conducted, the type of study, the intervention and placebo groups, the frequency of acupuncture or the dosage of pharmaceutical interventions (mg/day), the duration of intervention, the sample size, the 
mean and standard deviation of the glucose metabolism outcomes, and the mean and standard deviation of the lipid profile outcomes. Microsoft Excel with standard spreadsheets was used for data extraction.

2.5. Quality Evaluation. The risk of bias among the included studies was evaluated using the tool developed by the Cochrane Collaboration [30]. The results were cross-referenced, and any disagreements were resolved by discussion or consultation with a third evaluator with rich experience.

2.6. Outcome Measures. The primary outcome of interest was HOMA-IR. The secondary outcomes were fasting plasma glucose (FPG), fasting plasma insulin (FINS), $2 \mathrm{~h}$ fasting plasma glucose (2hFPG), $2 \mathrm{~h}$ fasting insulin (2hFINS), TC, TG, HDL-C, LDL-C, body mass index (BMI), waist-to-hip ratio (WHR), and the Ferriman-Gallwey score (FGS). The safety indicator was any adverse event.

2.7. Data Analysis. The quantitative analysis was carried out using the Cochrane Collaboration software RevMan 5.3.5. If the included studies used the same measurement scales, the continuous variables were described using the mean difference (MD) and 95\% confidence intervals (CIs). For the heterogeneity test, the chi-square test was performed first, and based on those results, the estimates of heterogeneity $\left(I^{2}\right)$ were applied. A fixed-effect model was used when the $I^{2}$ was $\leq 50 \%$ and the $P$ value was $\geq 0.1$, and a random-effect model was applied when the $I^{2}$ was $>50 \%$ or the $P$ value was $<0.10$. If heterogeneity was high, the source of heterogeneity was explored, and subgroup analysis or sensitivity analysis was performed to investigate the stability of the metaanalysis.

\section{Results}

3.1. Literature Search and Screening Flowchart. A flow chart of the study selection is shown in Figure 1. In all, 1077 articles were retrieved in our initial search. After removing duplications and screening the titles and abstracts, we obtained 39 full-text articles. Finally, 10 studies were included in the systematic review and meta-analysis.

\subsection{Characteristics of the Included Literature. A total of 737} patients were included in the 10 RCTs [31-40]. All participants were diagnosed with PCOS according to Rotterdam criteria [41], and they were treated with acupuncture alone or with placebo or metformin. The main acupoints used in these studies included CV3, RN4, RN6, ST25, ST28, ST36, SP9, and SP6. The specific features of these studies are summarized in Table 1.

3.3. Quality Evaluation of the Articles. Among these 10 studies, the patients were randomized by using a random number table in six studies [31,33-35, 37, 38], while the remaining four studies only mentioned "random" or "randomization" without describing the specific randomization methods. Only three articles described allocation concealment [31, 33, 40]. For participant and personnel blinding, five trials were at low risk of bias [31, $32,38-40]$, and the remaining studies [33-37] were given a high risk of bias due to the loss of blinding during implementation. Measurements were generally made by third parties other than the researchers, so the blinding of outcome assessment was defined as low risk. Two articles failed to describe the missing data, while in the remaining eight articles, the number of patients in all randomized groups was consistent with the number of subjects in the statistical analysis. The articles reported both the glucose metabolism and lipid profile indicators. The quality of the literature included in our analysis was average, and the details of the evaluation are shown in Figure 2.

\subsection{Results of the Meta-Analysis}

3.4.1. General Indicators. All included studies compared BMI and WHR, while only five studies reported FGS. There was evidence for a decrease in BMI in the acupuncture groups versus the control groups ( $\mathrm{MD}=-1.47,95 \% \mathrm{CI}$ $[-2.35$ to -0.58$], P<0.001)$. The overall effect showed a significant improvement in WHR between the groups $(\mathrm{MD}=-0.04,95 \%$ CI $[-0.06,-0.02], P<0.001)$. No significant difference was observed in FGS. The results of the meta-analysis are shown in Figure 3.

3.4.2. Glucose Metabolism Indicators. Nine articles reported HOMA-IR and eight articles reported FPG and FINS, but only four articles reported 2hFPG and only two reported $2 \mathrm{hFINS}$. The pooled results from nine studies showed a significant difference in HOMA-IR in the acupuncture groups compared with the control groups ( $\mathrm{MD}=-0.22,95 \%$ CI $[-0.41,-0.02], P=0.03)$. The pooled analysis showed a decrease in FPG in the acupuncture group ( $\mathrm{MD}=-0.38 ; 95 \%$ CI $[-0.70,-0.07], P=0.02)$. There was an improvement in FINS between the groups $(\mathrm{MD}=-0.99,95 \% \mathrm{CI}[-2.03,0.04]$, $P=0.06$ ), but this was not statistically significant. No significant differences were observed for the other outcomes. The results of the meta-analysis are shown in Figure 4.

3.4.3. Lipid Profile. Six studies with 370 participants were included to compare TC, TG, LDL-C, and HDL-C between groups. There was no significant difference in TC or HDL-C between the acupuncture and control groups, but a significant decrease was observed in TG in the acupuncture group $(\mathrm{MD}=-0.26,95 \% \mathrm{CI}[-0.48,-0.04], P=0.02)$. The results of the meta-analysis are shown in Figure 5.

3.4.4. Adverse Events. Six of the studies reported the presence of adverse events. Of these, two studies [31, 40] reported that there were no adverse events, while the remaining four studies [33, 36-38] reported gastrointestinal problems in the metformin groups such as nausea, vomiting, mild diarrhea, slight dizziness, or weakness. There were no adverse events found in the acupuncture groups of these 


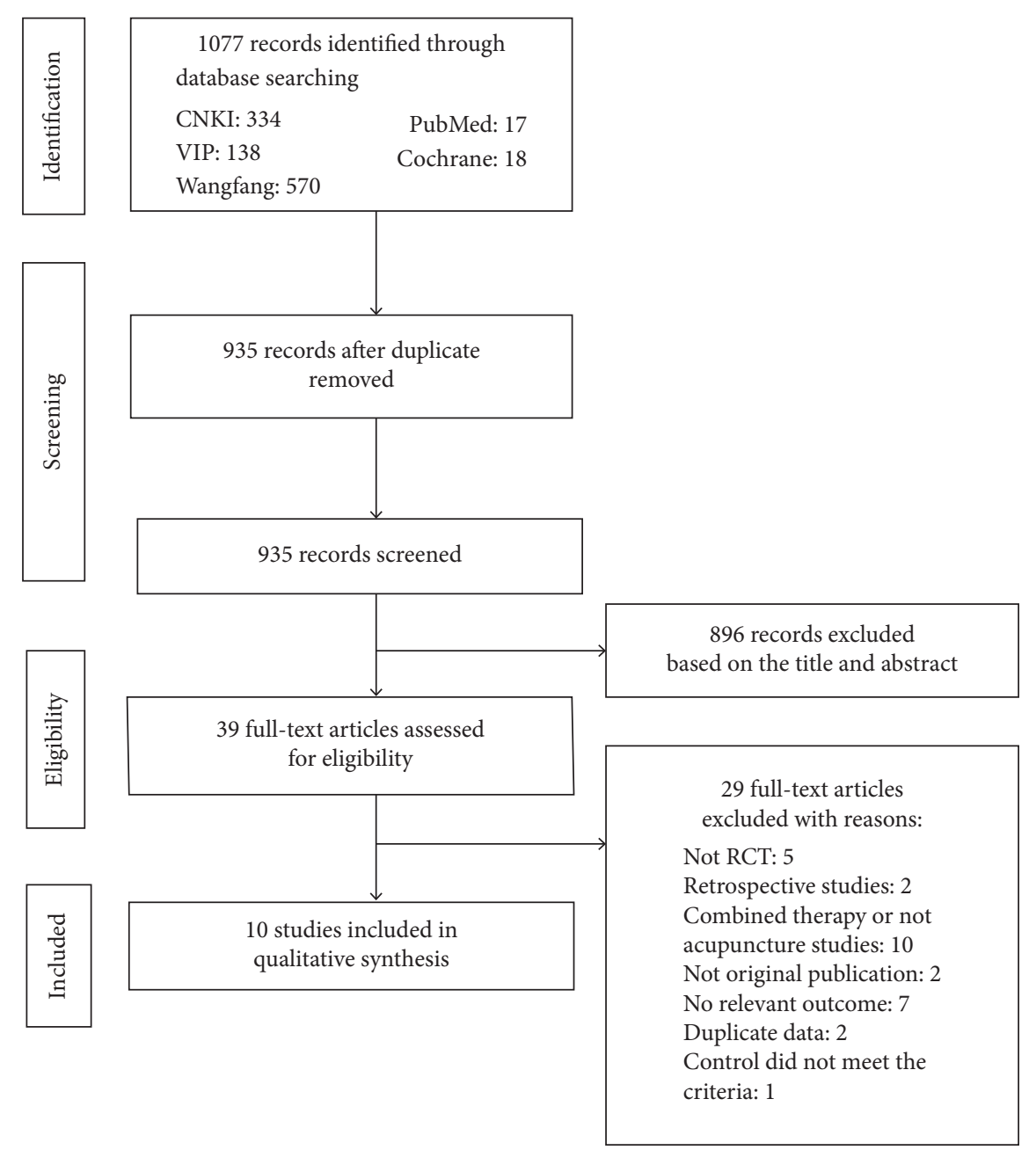

FIGURE 1: Literature search and screening flowchart.

studies except for one study [37] in which one patient had mild bleeding at the site of needling.

\section{Discussion}

4.1. Principle Findings. The objective of this review was to summarize and evaluate the use of acupuncture to improve glucose metabolism and lipid profiles in patients with PCOS. Overall, we found that acupuncture was closely associated with decreased BMI, WHR, FPG, and HOMA-IR and that acupuncture could significantly improve HOMA-IR and the level of fasting glucose in patients with PCOS, which confirms previous reports $[27,28]$. It is also reported that acupuncture can decrease BMI and WHR [28, 42, 43]. FINS, $2 \mathrm{hFPG}$, and $2 \mathrm{hFINS}$ were reduced in the acupuncture groups, but these differences were not significantly different. The results of the lipid profile were reported in a few studies, and we found that acupuncture significantly improved TG levels, while the differences in TC, LDL-C, and HDL-C were not significant. Acupuncture seems to be associated with a few adverse events, and the reported adverse events, such as bleeding, were mild and transient, demonstrating that acupuncture is safe and reliable. Thus, we conclude that acupuncture, compared with standard therapy, is more effective and safer in improving glucose metabolism and insulin sensitivity in patients with PCOS.

4.2. Limitations. First, this meta-analysis only included clinical studies comparing acupuncture alone versus placebo (placebo or sham acupuncture) or standard therapy. Studies on other combined therapies were not included because the combined therapies are more complex than monotherapy. Indeed, if acupuncture alone can achieve a curative effect without significant cost or time, then additional therapies might not be necessary. Second, there are certain heterogeneities among these studies. On the one hand, PCOS itself is heterogeneous by nature in terms of its clinical and biochemical features, and different distributions of ethnicity and age contribute to different manifestations of PCOS [44]. On the other hand, the disease severity and the dosages of metformin differed among the studies. Finally, some of the included studies did not describe the specific randomization method or did not adopt blinding. Therefore, these studies have just an average methodological quality. 
TABle 1: Features of the included studies.

\begin{tabular}{|c|c|c|c|c|c|c|}
\hline Study ID & $\begin{array}{l}\text { Sample } \\
\text { size }\end{array}$ & $\begin{array}{l}\text { Course of } \\
\text { PCOS }\end{array}$ & $\begin{array}{c}\text { Participant } \\
\text { characteristics }\end{array}$ & Age & Treatment versus control & Outcome \\
\hline $\begin{array}{l}\text { Stener- } \\
\text { Victorin, } \\
2009\end{array}$ & 15 & & & $\begin{array}{c}\mathrm{T}: \\
29.9 \pm 4.5 \\
\mathrm{C}: \\
30.4 \pm 5.5\end{array}$ & $\begin{array}{c}\text { Electroacupuncture group: the needles were } \\
\text { inserted into acupoints including CV3, RN6, } \\
\text { bilateral ST29, SP6, and LI4 (or PC6 } \\
\text { alternatively every second time) and retained } \\
\text { for } 30 \text { min. The procedure was administered } \\
\text { two times a week for } 2 \text { weeks, once a week for } 6 \\
\text { weeks, and then once every second week for } 8 \\
\text { weeks, giving a total of } 14 \text { treatments over } 16 \\
\text { weeks. Control group: this group was given } \\
\text { information about the importance of physical } \\
\text { activity and a healthy diet. }\end{array}$ & $\begin{array}{l}\text { BMI, WHR, FGS, FPG, } \\
\text { FINS, HOMA-IR, TC, } \\
\text { TG, LDL-C, HDL-C }\end{array}$ \\
\hline Zheng, 2012 & 86 & $\begin{array}{c}\mathrm{T}: \\
3.5 \pm 2.8 \\
\mathrm{C}: \\
3.6 \pm 2.2\end{array}$ & $\begin{array}{l}\text { Obesity-type } \\
\text { PCOS }\end{array}$ & $\begin{array}{c}\mathrm{T}: \\
26.5 \pm 3.0 \\
\text { C: } \\
24.9 \pm 4.9\end{array}$ & $\begin{array}{l}\text { Acupuncture group: the needles were inserted } \\
\text { into acupoints including RN4, RN6, RN10, } \\
\text { CV12, ST21, ST25, and ST28 and retained for } \\
30 \text { min. The procedure was administered twice } \\
\text { a week for } 6 \text { months. Control group: } \\
\text { metformin was taken with food for } 6 \text { months. } \\
\text { In the first week of the study, patients received } \\
250 \mathrm{mg} \text { three times daily; thereafter, the } \\
\text { metformin dose was } 500 \mathrm{mg} \text { three times daily. } \\
\text { Acupuncture group the needles were inserted }\end{array}$ & $\begin{array}{c}\text { BMI, WHR, FGS, FGP, } \\
\text { FINS, 2hFPG, 2hFINS, } \\
\text { HOMA-IR, TC, TG, } \\
\text { LDL-C, HDL-C }\end{array}$ \\
\hline Yao, 2018 & 97 & $\begin{array}{c}\mathrm{T}: \\
3.1 \pm 0.9 \\
\mathrm{C}: \\
3.2 \pm 0.8\end{array}$ & $\begin{array}{l}\text { Obesity-type } \\
\text { PCOS }\end{array}$ & $\begin{array}{c}\mathrm{T}: \\
27.8 \pm 4.8 \\
\mathrm{C}: \\
28.2 \pm 4.5\end{array}$ & $\begin{array}{l}\text { into acupoints including RN17, CV12, RN4, } \\
\text { bilateral BL18, ST25, RN19, ST36, LR14, SP6, } \\
\text { and LR3 and retained for } 30 \text { min. The } \\
\text { procedure was administered three times a } \\
\text { week for } 6 \text { months. Control group: metformin } \\
\text { was taken at } 500 \text { mg three times daily with } \\
\text { food for } 6 \text { months. }\end{array}$ & $\begin{array}{c}\text { BMI, WHR, FGS, 2hFPG, } \\
\text { HOMA-IR }\end{array}$ \\
\hline Kong, 2015 & 55 & & $\begin{array}{l}\text { Obesity-type } \\
\text { PCOS }\end{array}$ & $\begin{array}{c}\mathrm{T}: \\
28.1 \pm 3.8 \\
\mathrm{C}: \\
27.8 \pm 3.4\end{array}$ & $\begin{array}{l}\text { Electroacupuncture group: the needles were } \\
\text { inserted into acupoints including DU20, CV3, } \\
\text { RN6, bilateral LI4, SP6, ST29, and SP9 and } \\
\text { retained for } 30 \text { min. The needles were then } \\
\text { inserted into acupoints including DU20, CV3, } \\
\text { bilateral ST29, PC6, SP6, ST25, and LR3 and } \\
\text { retained for } 30 \text { min. The procedure was } \\
\text { performed two or three times a week for a total } \\
\text { of } 32 \text { sessions. Control group: Sham } \\
\text { acupuncture was used, and virtual } \\
\text { electroacupuncture was used at the acupoint. } \\
\text { Acupuncture group: the needles were inserted }\end{array}$ & $\begin{array}{l}\text { BMI, WHR, FGP, FINS, } \\
\text { HOMA-IR, TC, TG, } \\
\text { LDL-C, HDL-C }\end{array}$ \\
\hline Peng, 2017 & 100 & $\begin{array}{c}\mathrm{T}: \\
2.5 \pm 0.8 \\
\mathrm{C}: \\
2.4 \pm 0.9\end{array}$ & & $\begin{array}{c}\mathrm{T}: \\
28.6 \pm 3.8 \\
\mathrm{C}: \\
28.8 \pm 3.3\end{array}$ & $\begin{array}{c}\text { ST25 and retained for } 30 \text { min. The procedure } \\
\text { was administered three times a week for } 3 \\
\text { months. Control group: Sham acupuncture } \\
\text { was administered three times a week for } 3 \\
\text { months. }\end{array}$ & BMI, WHR \\
\hline $\mathrm{Li}, 2014$ & 100 & & & & $\begin{array}{c}\text { Acupuncture group: the needles were inserted } \\
\text { into acupoints including CV3, RN4, bilateral } \\
\text { ST36, SP6, KI7, and RN6 and retained for } \\
30 \text { min. The procedure was performed every } \\
\text { day except during the menstrual period. } \\
\text { Control group: both sham acupuncture and } \\
\text { metformin were used. Metformin was taken as } \\
\text { a single tablet with food three times daily for } 6 \\
\text { months. }\end{array}$ & $\begin{array}{l}\text { BMI, WHR, FGS, FGP, } \\
\text { FINS, HOMA-IR, TC, } \\
\text { TG, LDL-C, HDL-C }\end{array}$ \\
\hline
\end{tabular}


TABLE 1: Continued.

\begin{tabular}{|c|c|c|c|c|c|c|}
\hline Study ID & $\begin{array}{l}\text { Sample } \\
\text { size }\end{array}$ & $\begin{array}{l}\text { Course of } \\
\text { PCOS }\end{array}$ & $\begin{array}{c}\text { Participant } \\
\text { characteristics }\end{array}$ & Age & Treatment versus control & Outcome \\
\hline Cai, 2016 & 50 & & $\begin{array}{l}\text { Obesity-type } \\
\text { PCOS }\end{array}$ & & $\begin{array}{c}\text { Acupuncture group: the needles were inserted } \\
\text { into acupoints including CV12, ST21, ST25, } \\
\text { GB26, RN6, RN4, ST28, SP10, ST34, ST36, } \\
\text { ST37, and SP6 and retained for } 30 \text { min. The } \\
\text { procedure was performed three times a week } \\
\text { for } 3 \text { months. Control group: metformin was } \\
\text { taken at } 500 \text { mg three times daily with food for } \\
6 \text { months. }\end{array}$ & $\begin{array}{c}\text { BMI, WHR, FGP, FINS, } \\
\text { HOMA-IR }\end{array}$ \\
\hline Lai, 2012 & 120 & $\begin{array}{c}\text { T: } \\
2.5 \pm 0.6 \\
\text { C: } \\
2.5 \pm 0.7\end{array}$ & & $\begin{array}{c}\mathrm{T}: \\
26.7 \pm 2.7 \\
\mathrm{C}: \\
26.5 \pm 2.7\end{array}$ & $\begin{array}{l}\text { Acupuncture group: the needles were inserted } \\
\text { into acupoints including CV12, RN10, RN6, } \\
\text { RN4, ST25, and ST } 28 \text { and retained for } 30 \text { min. } \\
\text { The procedure was performed once every } 3 \\
\text { days for } 4 \text { months. Control group: metformin } \\
\text { was taken at } 500 \text { mg three times daily with } \\
\text { food for } 4 \text { months. }\end{array}$ & $\begin{array}{l}\text { BMI, WHR, FGP, FINS, } \\
\text { 2hFPG, 2hFINS, HOMA- } \\
\text { IR }\end{array}$ \\
\hline Guo, 2014 & 38 & & PCOS-IR & $\begin{array}{c}\mathrm{T}: \\
27.8 \pm 3.2 \\
\mathrm{C}: \\
29.3 \pm 2.9\end{array}$ & $\begin{array}{l}\text { Electropuncture group: the needles were } \\
\text { inserted into acupoints including CV3, RN6, } \\
\text { DU20, bilateral ST29, SP6, SP9, and LI4 and } \\
\text { retained for } 30 \text { min. The needles were then } \\
\text { inserted into acupoints including CV3, RN6, } \\
\text { DU20, bilateral ST25, ST29, SP6, LR3, and } \\
\text { PC6 and retained for } 30 \text { min. The procedure } \\
\text { was performed once every } 2 \text { days for } 2 \text { months. } \\
\text { Control group: Sham acupuncture was used } \\
\text { and retained for } 30 \text { min. The procedure was }\end{array}$ & $\begin{array}{l}\text { BMI, WHR, FGS, FGP, } \\
\text { FINS, HOMA-IR, TC, } \\
\text { TG, LDL-C, HDL-C }\end{array}$ \\
\hline Gu, 2019 & 76 & $\begin{array}{c}\mathrm{T}: \\
4.6 \pm 3.6 \\
\mathrm{C}: \\
4.3 \pm 3.3\end{array}$ & PCOS-IR & $\begin{array}{c}\mathrm{T}: \\
27.0 \pm 4.5 \\
\mathrm{C}: \\
28.6 \pm 4.0\end{array}$ & $\begin{array}{l}\text { performed once every } 2 \text { days for } 2 \text { months. } \\
\text { Electroacupuncture group: the needles were } \\
\text { inserted into acupoints including CV3, RN6, } \\
\text { DU20, bilateral ST29, SP6, SP9, and LI4 and } \\
\text { retained for } 30 \text { min. The needles were then } \\
\text { inserted into acupoints including CV3, RN6, } \\
\text { DU20, bilateral ST25, ST29, SP6, LR3, and } \\
\text { PC6, and retained for } 30 \text { min. The procedure } \\
\text { was performed twice a week for a total of } 32 \\
\text { sessions. Control group: Sham acupuncture } \\
\text { was used and retained for } 30 \text { min. The } \\
\text { procedure was performed twice a week for a } \\
\text { total of } 32 \text { sessions. }\end{array}$ & $\begin{array}{c}\text { BMI, WHR, FGP, 2hFPG, } \\
\text { FINS, 2hFINS, HOMA- } \\
\text { IR, TC, TG, LDL-C, } \\
\text { HDL-C }\end{array}$ \\
\hline
\end{tabular}

BMI: body mass index; WHR: waist-to-hip ratio; FGS: Ferriman-Gallwey score; FPG: fasting plasma glucose; 2hFPG: 2 h fasting plasma glucose; FINS: fasting insulin; 2hFINS: $2 \mathrm{~h}$ fasting insulin; HOMA-IR: homeostasis model assessment of insulin resistance; TC: serum total cholesterol; TG: triglyceride; LDL-C: low-density lipoprotein cholesterol; HDL-C: high-density lipoprotein cholesterol.

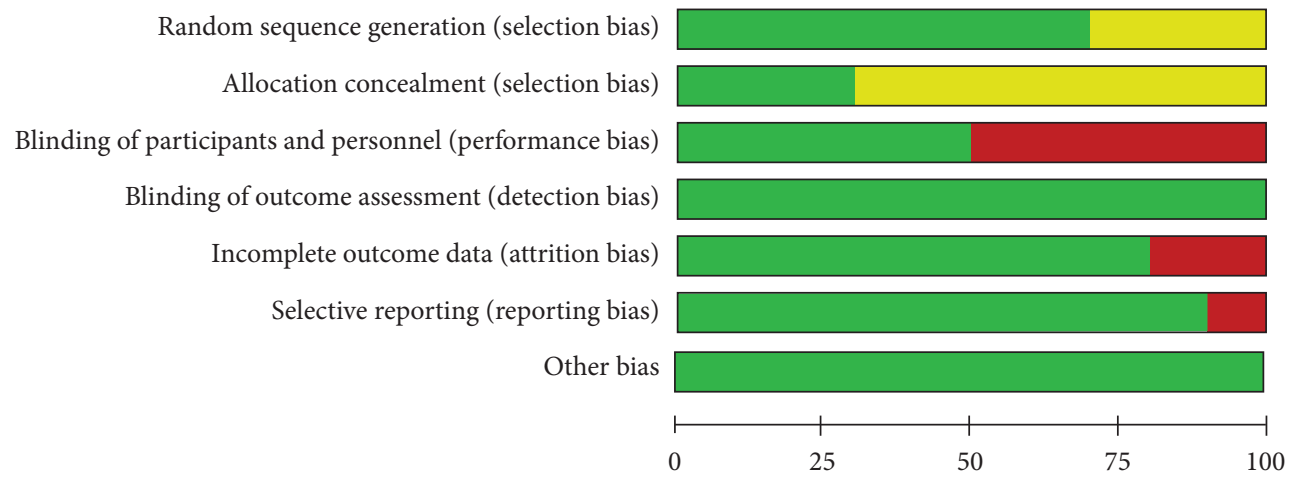

$(\%)$

Low risk of bias

High risk of bias

Unclear risk of bias

FIGURE 2: Evaluation of the risk biases of the included studies. 


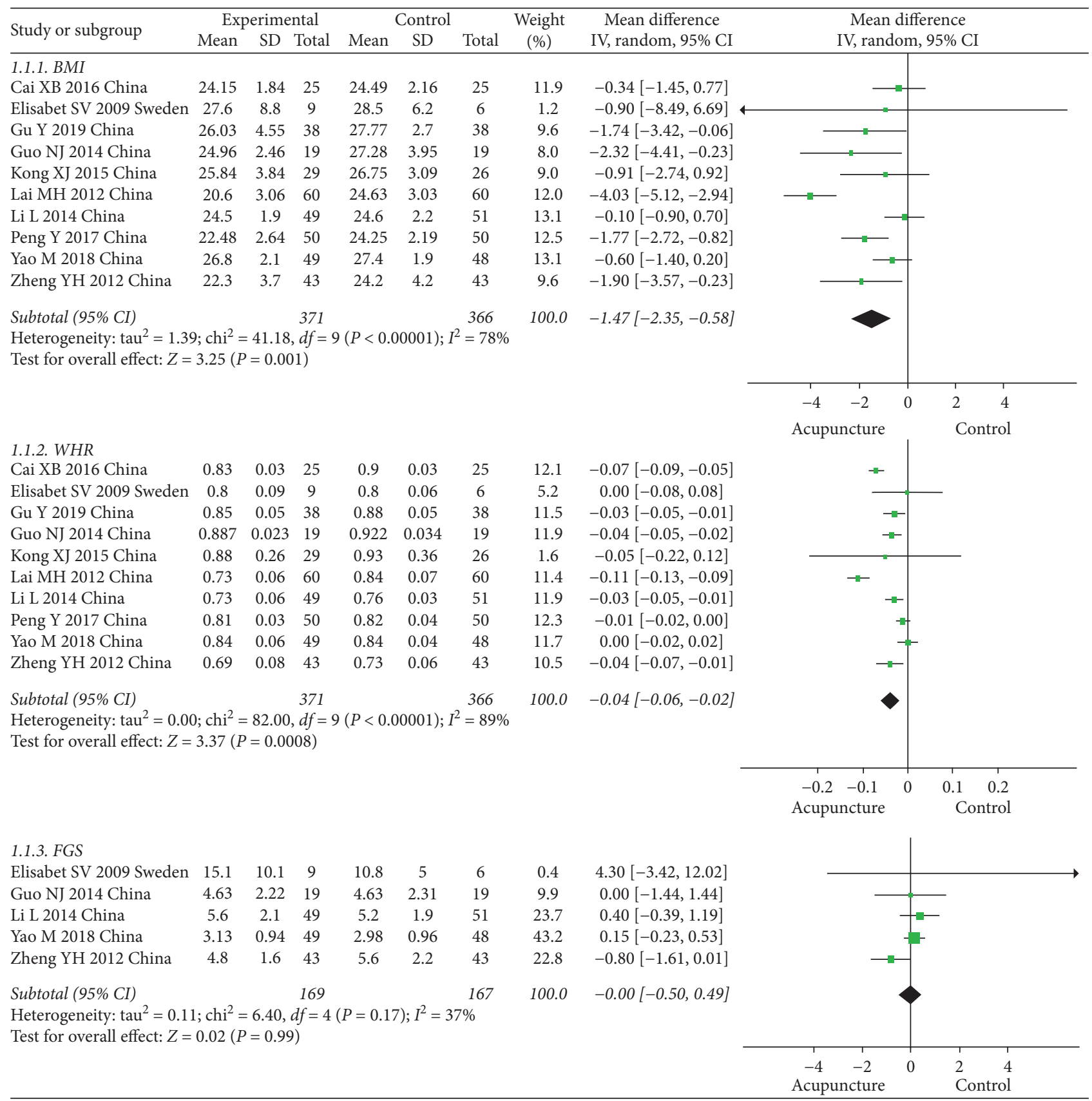

FIgure 3: Comparison of the BMI, WHR, and FGS between the acupuncture and control groups in the treatment of PCOS.

4.3. Implications for Clinical Practice and Further Research. The results of this meta-analysis suggest that acupuncture alone has better efficacy than control interventions. Because traditional Chinese medicine departments have been established in most maternity hospitals and general hospitals in China, it is easy and convenient to apply such treatments. As a safe and simple therapy, acupuncture might be an alternative or a good adjunct therapy for PCOS, especially for overweight patients and patients with IR. However, we have no information on how long the effect of acupuncture might last. Thus, more long-term follow-up studies are needed to examine the effectiveness of acupuncture in improving insulin resistance and depressing BMI and WHR and to assess the sustainability of their effects.

Several meta-analyses have been performed regarding the effectiveness of acupuncture for treating PCOS, specifically focusing on rates of ovulation, pregnancy, and live birth [45-47], but articles about how acupuncture affects metabolic-related indexes in patients with PCOS, particularly lipid profiles, are lacking. 


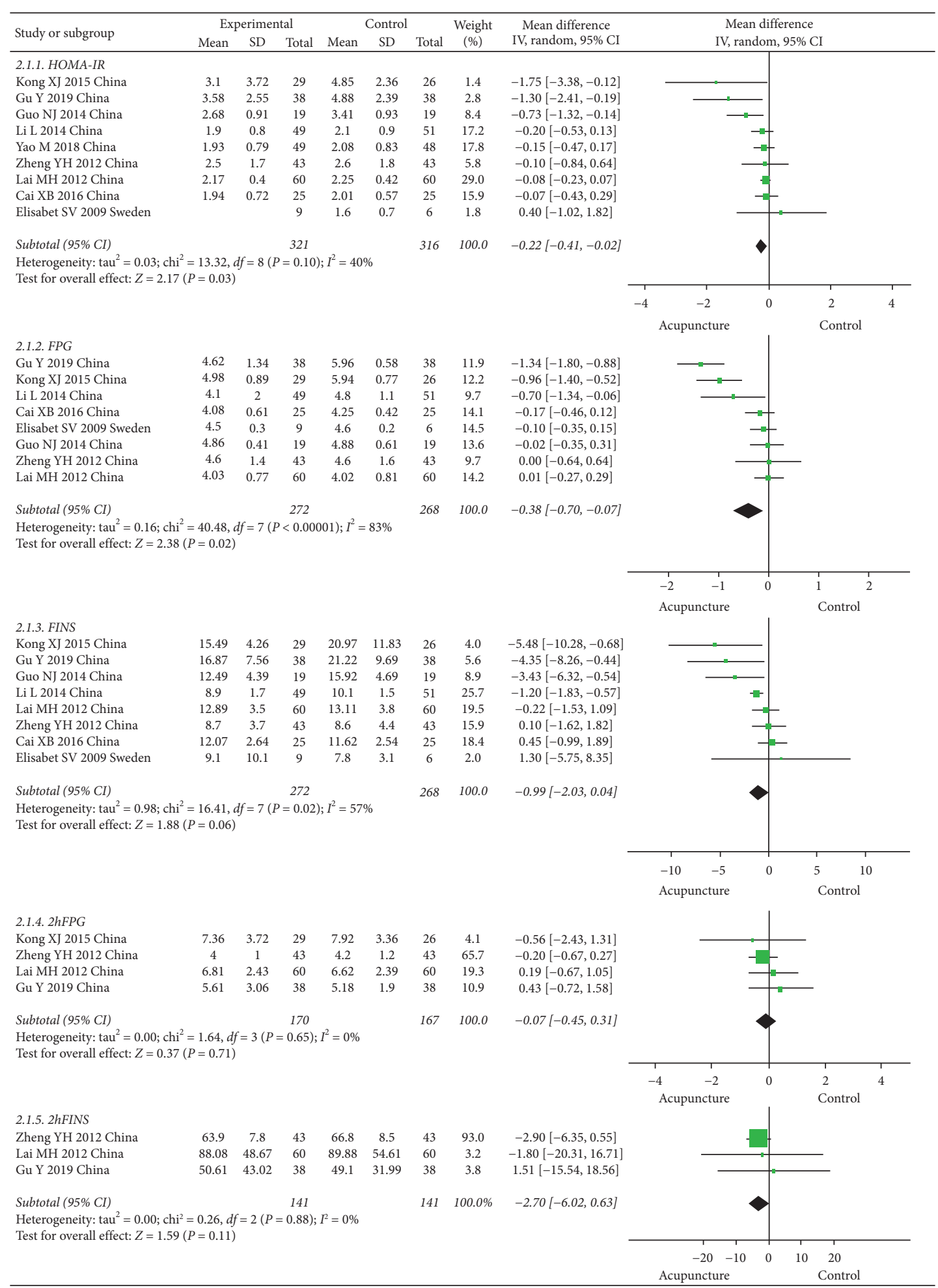

FIgURE 4: Comparison of glucose metabolism indicators between the acupuncture and control groups in the treatment of PCOS. 


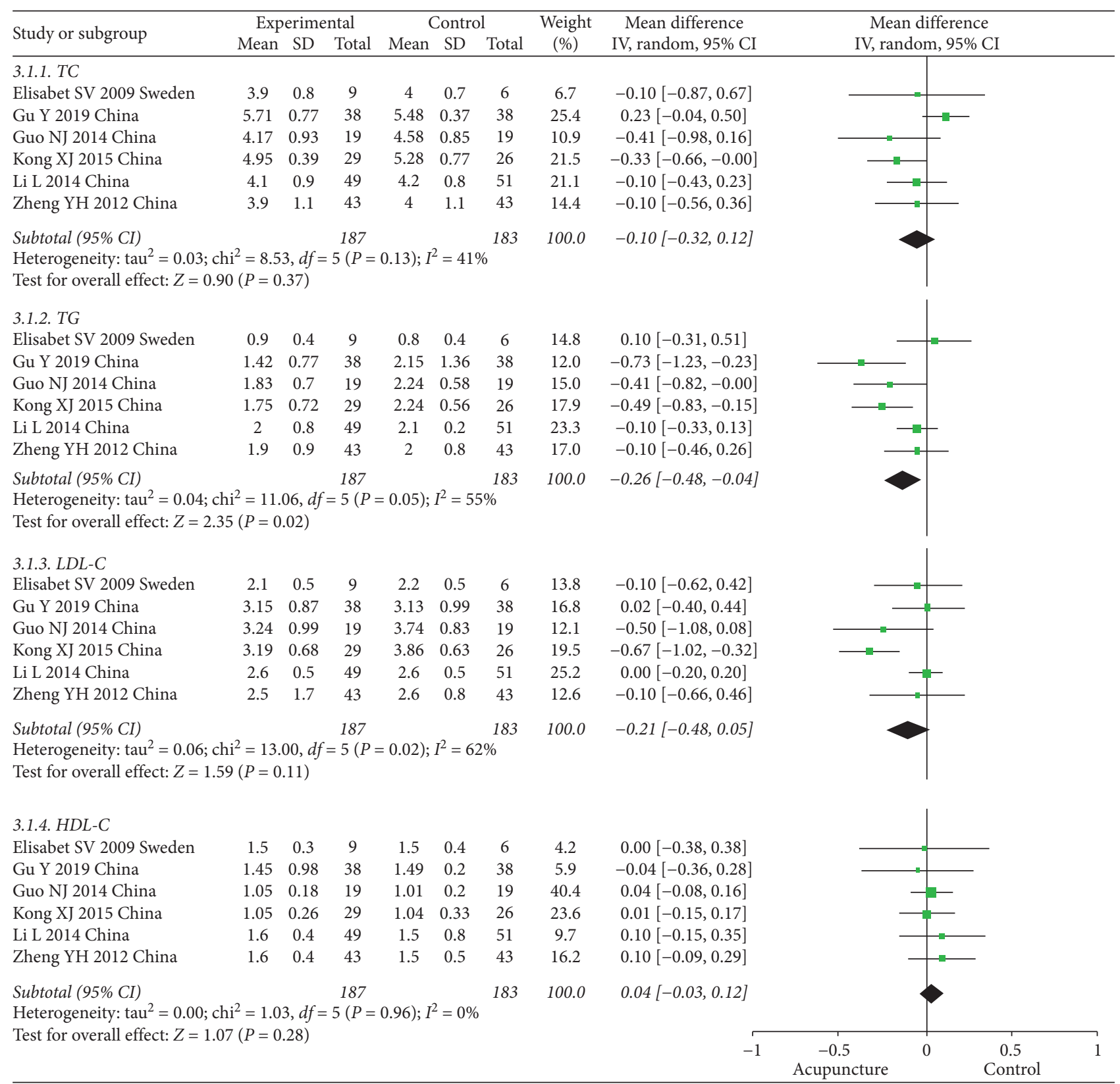

FIGURE 5: Comparison of lipid profiles between the acupuncture and control groups in the treatment of PCOS.

\section{Conclusion}

There is insufficient evidence to support that acupuncture can improve lipid profiles except for TG levels. However, this review of 10 RCTs shows that acupuncture could improve BMI and WHR as well as HOMA-IR in patients with PCOS. The included studies are inconclusive because of their moderate level of evidence, and further largescale, long-term RCTs with rigorous methodological standards are still warranted.

\section{Disclosure}

RZ and PQ are the co-first authors.

\section{Conflicts of Interest}

The authors declare no conflicts of interest.

\section{Authors' Contributions}

JL and HM contributed equally to this work. JL conceptualized and designed the study. RZ drafted the manuscript. $\mathrm{RZ}$ and PQ collected and analyzed the data. $\mathrm{MH}, \mathrm{JS}, \mathrm{MH}$, and HM reviewed the protocol for important intellectual content and revised the manuscript critically. JL and HM sought funding. All authors contributed to the further editing of the manuscript and approved the final version of the manuscript accepted for publication. Ruqun Zheng and Peng Qing contributed equally. 


\section{Acknowledgments}

This work was supported by the National Natural Science Foundation of China, under Grant 81804137, and the Science and Technology Planning Project of Guangdong Province, under Grants 2017A020213004 and 2014A020221060 (Dr. J. Li).

\section{References}

[1] D. Lizneva, L. Suturina, W. Walker, S. Brakta, L. GavrilovaJordan, and R. Azziz, "Criteria, prevalence, and phenotypes of polycystic ovary syndrome," Fertility and Sterility, vol. 106, no. 1 , pp. 6-15, 2016.

[2] R. Azziz, E. Carmina, D. Dewailly et al., "The androgen excess and PCOS society criteria for the polycystic ovary syndrome: the complete task force report," Fertility and Sterility, vol. 91, no. 2, pp. 456-488, 2009.

[3] S. Franks, M. I. McCarthy, and K. Hardy, "Development of polycystic ovary syndrome: involvement of genetic and environmental factors," International Journal of Andrology, vol. 29, no. 1, pp. 286-290, 2006.

[4] V. De Leo, M. C. Musacchio, V. Cappelli, M. G. Massaro, G. Morgante, and F. Petraglia, "Genetic, hormonal and metabolic aspects of PCOS: an update," Reproductive Biology and Endocrinology, vol. 14, p. 38, 2016.

[5] "American association of clinical endocrinologists position statement on metabolic and cardiovascular consequences of polycystic ovary syndrome," Endocrine Practice, vol. 21, pp. 126-134, 2005.

[6] S. Franks, "When should an insulin sensitizing agent be used in the treatment of polycystic ovary syndrome?" Clinical Endocrinology, vol. 74, no. 2, pp. 148-151, 2011.

[7] C. Celik, N. Tasdemir, R. Abali, E. Bastu, and M. Yilmaz, "Progression to impaired glucose tolerance or type 2 diabetes mellitus in polycystic ovary syndrome: a controlled follow-up study," Fertility and Sterility, vol. 101, no. 4, pp. 1123-1128, 2014.

[8] B. Bhagavath, W. Vitek, J. Queenan, and K. Hoeger, "Metformin and other insulin sensitizers in polycystic ovary syndrome," Seminars in Reproductive Medicine, vol. 32, no. 4, pp. 323-330, 2014.

[9] D. A. Ehrmann, R. B. Barnes, R. L. Rosenfield, M. K. Cavaghan, and J. Imperial, "Prevalence of impaired glucose tolerance and diabetes in women with polycystic ovary syndrome," Diabetes Care, vol. 22, no. 1, pp. 141-146, 1999.

[10] R. S. Legro, A. R. Kunselman, W. C. Dodson, and A. Dunaif, "Prevalence and predictors of risk for type 2 diabetes mellitus and impaired glucose tolerance in polycystic ovary syndrome: a prospective, controlled study in 254 affected Women1," The Journal of Clinical Endocrinology \& Metabolism, vol. 84, no. 1, pp. 165-169, 1999.

[11] D. Wiltgen and P. M. Spritzer, "Variation in metabolic and cardiovascular risk in women with different polycystic ovary syndrome phenotypes," Fertility and Sterility, vol. 94, no. 6, pp. 2493-2496, 2010.

[12] L. Mannerås, I. H. Jonsdottir, A. Holmäng, M. Lönn, and E. Stener-Victorin, "Low-frequency electro-acupuncture and physical exercise improve metabolic disturbances and modulate gene expression in adipose tissue in rats with dihydrotestosterone-induced polycystic ovary syndrome," Endocrinology, vol. 149, no. 7, pp. 3559-3568, 2008.

[13] D. Panidis, K. Tziomalos, E. Papadakis, C. Vosnakis, P. Chatzis, and I. Katsikis, "Lifestyle intervention and antiobesity therapies in the polycystic ovary syndrome: impact on metabolism and fertility," Endocrine, vol. 44, no. 3, pp. 583-590, 2013.

[14] E. Seli and A. J. Duleba, "Treatment of PCOS with metformin and other insulin-sensitizing agents," Current Diabetes Reports, vol. 4, no. 1, pp. 69-75, 2004.

[15] N. Naderpoor, S. Shorakae, B. de Courten, M. L. Misso, L. J. Moran, and H. J. Teede, "Metformin and lifestyle modification in polycystic ovary syndrome: systematic review and meta-analysis," Human Reproduction Update, vol. 21, no. 5, pp. 560-574, 2015.

[16] L. C. Morley, T. Tang, E. Yasmin, R. J. Norman, and A. H. Balen, "Insulin-sensitising drugs (metformin, rosiglitazone, pioglitazone, D-chiro-inositol) for women with polycystic ovary syndrome, oligo amenorrhoea and subfertility," The Cochrane Database of Systematic Reviews, vol. 11, 2017.

[17] S. K. Graff, F. M. Mario, P. Ziegelmann, and P. M. Spritzer, "Effects of orlistat vs. Metformin on weight loss-related clinical variables in women with PCOS: systematic review and meta-analysis," International Journal of Clinical Practice, vol. 70, no. 6, pp. 450-461, 2016.

[18] M. O. Goodarzi and M. Bryer-Ash, "Metformin revisited: Reevaluation of its properties and role in the pharmacopoeia of modern antidiabetic agents," Diabetes, Obesity and Metabolism, vol. 7, no. 6, pp. 654-665, 2005.

[19] E. Stener-Victorin, E. Jedel, and L. Mannerås, “Acupuncture in polycystic ovary syndrome: current experimental and clinical evidence," Journal of Neuroendocrinology, vol. 20, no. 3, pp. 290-298, 2008.

[20] W. L. Bennett, L. M. Balfe, and J. M. Faysal, "AHRQ's comparative effectiveness research on oral medications for type 2 diabetes: a summary of the key findings," Journal of Managed Care Pharmacy, vol. 18, no. 1 Supp A, pp. 1-22, 2012.

[21] T. J. Kaptchuk, "Methodological issues in trials of acupuncture," JAMA, vol. 285, pp. 1015-1016, 2001.

[22] R.-Q. Zhang, J. Tan, F.-Y. Li, Y.-H. Ma, L.-X. Han, and X.-L. Yang, "Acupuncture for the treatment of obesity in adults: a systematic review and meta-analysis," Postgraduate Medical Journal, vol. 93, no. 1106, pp. 743-751, 2017.

[23] X. Kang, L. Jia, Y. Li, and Xu Zhang, "Acupuncture attenuates hyperglycaemia and improves ovarian function in female rats subjected to continuous light exposure," Journal of the British Medical Acupuncture Society, vol. 35, no. 5, pp. 352-359, 2017.

[24] S. L. Chang, J. G. Lin, T. C. Chi, I. M. Liu, and J. T. Cheng, "An insulin-dependent hypoglycaemia induced by electroacupuncture at the zhongwan (CV12) acupoint in diabetic rats," Diabetologia, vol. 42, no. 2, pp. 250-255, 1999.

[25] Y. Kang, M. Li, W. Yan, X. Li, J. Kang, and Y. Zhang, "Electroacupuncture alters the expression of genes associated with lipid metabolism and immune reaction in liver of hypercholesterolemia mice," Biotechnology Letters, vol. 29, no. 12, pp. 1817-1824, 2007.

[26] F. Liang and D. Koya, "Acupuncture: is it effective for treatment of insulin resistance?" Diabetes, Obesity \& Metabolism, vol. 12, no. 7, pp. 555-569, 2010.

[27] J. Pundir, D. Charles, L. Sabatini et al., "Overview of systematic reviews of non-pharmacological interventions in women with polycystic ovary syndrome," Human Reproduction Update, vol. 25, no. 2, pp. 243-256, 2019.

[28] J. Li, W. Wu, E. Stener-Victorin et al., "A prospective pilot study of the effect of acupuncture on insulin sensitivity in women with polycystic ovary syndrome and insulin resistance," Journal of the British Medical Acupuncture Society, vol. 38, no. 5, pp. 310-318, 2020. 
[29] M.-H. Lai, H.-X. Ma, J. Li, X.-H. Song, and H. Liu, "Effects of electroacupuncture on mRNA expressions of insulin-receptor substrates 1 and 2 in the endometrium of PCOS rats and insulin sensitivity," Zhongguo Zhong Xi Yi Jie He Za Zhi, vol. 36, no. 9, pp. 1082-1086, 2016.

[30] J. P. T. Higgins, D. G. Altman, P. Juni, D. Moher, A. D. Oxman, J. Savovic et al., “The Cochrane collaboration's tool for assessing risk of bias in randomised trials," $B M J$, vol. 343, no. 2, p. d5928, 2011.

[31] X. Sterne, Polycystic Ovary Syndrome Evaluation of Comprehensive Effects of Abnormal Glucose and Lipid Metabolism of Acupuncture Intervention, Nanjing University of Chinese Medicine, Nanjing, China, 2015.

[32] N. Guo, The Clinical Curative Effect of Acupuncture in Patients with Polycystic Ovary Syndrome with Insulin Resistance, Nanjing University of Chinese Medicine, Nanjing, China, 2014.

[33] Y.-H. Zheng, X.-H. Wang, M.-H. Lai, H. Yao, H. Liu, and H.-X. Ma, "Effectiveness of abdominal acupuncture for patients with obesity-type polycystic ovary syndrome: a randomized controlled trial," The Journal of Alternative and Complementary Medicine, vol. 19, no. 9, pp. 740-745, 2013.

[34] E. Stener-Victorin, E. Jedel, and Y. B. Sverrisdottir, "Lowfrequency electroacupuncture and physical exercise decrease high muscle sympathetic nerve activity in polycystic ovary syndrome," American Journal of Physiology-Regulatory, Integrative and Comparative Physiology, vol. 297, no. 2, pp. R387-R395, 2009.

[35] X. Janson, Y. Li, J. Wang, and J. Cao, "Clinical observation of electro-acupuncture and acupoint catgut-embedding therapy in the treatment of obese polycystic ovary syndrome," Guangming Journal of Chinese Medicine, vol. 31, no. 4, pp. 538-541, 2016.

[36] M.-H. Lai, H.-X. Ma, H. Liu, X.-H. Song, T. Ding, and Y.-H. Zheng, "Clinical observation of 60 cases of spleenkidney yang deficiency polycystic ovary syndrome treated with abdominal acupuncture," Jiangsu Journal of Traditional Chinese Medicine, vol. 44, no. 8, pp. 53-54, 2012.

[37] M. Yao, D. Ding, W. Zhou, W. Huang, and X. Xu, "Effect of acupuncture and moxibustion on patients' anxiety in treatment of obesity type polycystic ovary syndrome," Acta Chinese Medicine, vol. 33, no. 10, pp. 2043-2048, 2018.

[38] L. I. Li, M. O. Hui, B. WEN et al., "Clinical study of the acupuncture combined with metformin for infertility patients with obesity-type polycystic ovary syndrome," China Journal of Traditional Chinese Medicine and Pharmacy, vol. 29, no. 7, pp. 2115-2119, 2014.

[39] Y. Peng, J. Cong, H. Ni-na, C. Hui, L. Na, and Y. Xin-ming, "Acupuncture with resolving phlegm therapy in the treatment of polysystic ovary syndrome: evaluation clinical efficacy," Journal of Clinical Acupuncture and Moxibustion, vol. 33, no. 2, pp. 5-8, 2017.

[40] Y. GU, X. U. Xiao-feng, and X.-yan HE, "Evaluation the effect of acupuncture improves glycolipid metabolism disorder in patients with polycystic ovary syndrome," Lishizhen Medicine and Materia Medica Research, vol. 30, no. 10, pp. 2431-2433, 2019.

[41] ASRM-Sponsored PCOS Consensus Workshop Group, "Revised 2003 consensus on diagnostic criteria and long-term health risks related to polycystic ovary syndrome," Fertility and Sterility, vol. 81, pp. 19-25, 2004.

[42] H.-L. Zhang, Ze-J. Huo, H.-N. Wang et al., "Acupuncture ameliorates negative emotion in PCOS patients: a randomized controlled trial," Zhongguo Zhen Jiu, vol. 40, no. 4, pp. 385390, 2020.

[43] J. Xu and Yu Zuo, "Efficacy of acupuncture as adjunctive treatment on infertility patients with polycystic ovary syndrome," Zhongguo Zhen Jiu, vol. 38, no. 4, pp. 358-361, 2018.

[44] J. Johansson and E. Stener-Victorin, "Polycystic ovary syndrome: effect and mechanisms of acupuncture for ovulation induction," Evidence-Based Complementary and Alternative Medicine, vol. 2013, Article ID 762615, 16 pages, 2013.

[45] J. Wu, Di Chen, and N. Liu, "Effectiveness of acupuncture in polycystic ovary syndrome: a systematic review and metaanalysis of randomized controlled trials," Medicine, vol. 99, no. 22, Article ID e20441, 2020.

[46] J. Jo and J. Yoon, H. Lee, Acupuncture for polycystic ovarian syndrome: a systematic review and meta-analysis," Medicine, vol. 96, Article ID e7066, 23 pages, 2017.

[47] J. Jo and J. L. Yoon, "Effectiveness of acupuncture in women with polycystic ovarian syndrome undergoing in vitro fertilisation or intracytoplasmic sperm injection: a systematic review and meta-analysis," Journal of the British Medical Acupuncture Society, vol. 35, no. 3, pp. 162-170, 2017. 